\title{
Recent Progress in High Shear Wet Granulation Process
}

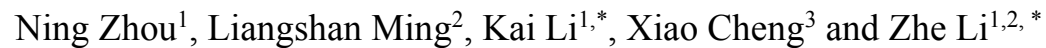 \\ ${ }^{1}$ Henan University of Chinese Medicine, Zhengzhou, Henan, 450008, China \\ ${ }^{2}$ Shanghai University of Traditional Chinese Medicine, Shanghai, 201203, China \\ ${ }^{3}$ Women and infants Hospital of Zhengzhou, Zhengzhou, 45000, China \\ ${ }^{*}$ Corresponding author
}

\begin{abstract}
High shear wet granulation is widely used in the pharmaceutical industry, however it is still difficult to predict the results of granulation and explain the relevant results. The paper reviews the three key processes in high shear granulation: wetting and nucleation, consolidation and growth, and breakage and attrition, combines with the corresponding models, the dimensionless parameters, regime maps and related mechanisms. Summarizes the basis and methods for judgment of granulation endpoint of final product. And proposed future direction of development of granulation and research ideas, which helps to solve the existing problems in the production and accelerate its development.
\end{abstract}

Keywords-review; high shear granulation; wetting and nucleation; consolidation and growth; and breakage and attrition

\section{INTRODUCTION}

Granulation is defined a process of size enlargement, that is to say, smaller powder particles are agglomerated into larger, semi-permanent or permanent aggregates in which original particles can still be distinguished. Meanwhile, the aggregates have better flowability and compressibility[1-2]. The process of granulation aims at particle growth, and the size of pharmaceutical agglomerates ranges from 0.1 to $2.0 \mathrm{~mm}$. The main purpose of granulation is to improve one or more powder properties, and they include the increasement of flowability, compressibility and bulk density, reduction the risk of size segregation, and dust formation, beautification of the appearance of the product, controlling the release of drugs, and the uniformity of accurate dosage[3]. Mixing, wetting, and granulation are accomplished in a single operation unit in high shear granulation (HSG), which saves on time and labor costs. It also simplifies the process of granulation. HSG is widely used in pharmaceutical industry for its many merits in recent 30 years[4], but rarely used in the field of traditional Chinese medicine (TCM), and the application in TCM still stays the stage of preliminary research of granulation process. Without uniform characterization methods of granulating design and process operation, pharmaceutical workers can't predict the granulation behavior and don't know how to change the formulation to obtain desired product. In pharmaceutical industry, pharmaceutical workers get the better formulation and process parameters with using a trial and error way, but the results are unsatisfied in most cases. Therefore, further study on the process of HSG is a key step in pharmaceutical industry, it can also promote its application in TCM. In this study, we describe the studies on different stages of granulation process in HSG and the properties of granulation product. This could promote modern research on TCM.

There are three distinct rate processes that occur in industrial granulators, they include wetting and nucleation, consolidation and growth, and breakage and attrition. During the stage of wetting and nucleation, most of the particles, which can be called powders, are wetting by granulation liquid uniformly to form initial nuclei. There are a large number of particle-impact behaviors that occur in the stage of consolidation and growth, the particle-impact behaviors may exist in the granules, or between granule and powder, or between granule and granulator. These behavior will make the particles merge and compaction, and promote the formation of granule. During the stage of breakage and attrition, the product of granulation is abrased and broken in the following operations such as drying. Breakage and attrition behavior may be caused by the impact behaviors. The corresponding dimensionless parameters and state diagram are proposed for different stages, and we can distinguish them by the torque value or particle size distribution[5].

\section{WETTING AND NUCLEATION}

The granulation liquid addition methods include pouring by a container, pumping by a constant flow pump and spraying by a atomization device. Different granulation liquid addition methods can affect the droplet size of liquid binder. Karin's research demonstrated that the droplet size of the binder liquid had great influence on agglomerate size and binder distribution in granulation[6]. Compared with pouring by a container, liquid binder sprayed into the mixer spreads more uniformly on the powder bed, and we will obtain desired granule with a narrower particle size distribution. This phenomenon can also avoid over-wetting during granulation.

During the stage of wetting, the liquid binder droplets impacting on a powder bed surface, the surface tension of the binder will act to pull powder particles together. The interactional behavior of the liquid binder droplets impacting on a powder bed surface will influence the properties of final granule significantly. The competing spreading-infiltration behavior can be observed in this case[7]. Drop penetration is driven by the Laplace Capillary Suction Pressure. In order to obtain desired granule with a narrower particle size distribution, the droplet must permeate into the powder bed quickly. While the drop penetration behavior on loosely 
packed porous powder beds is highly depend on the microstructure of the bed and quite complex. The Laplace Capillary Suction Pressure $P c$ is affected by liquid surface tension $\gamma$, pore size of the bed $R$ and the solid-liquid thermodynamic contact angle $\theta$, Eq. 1 .

$$
\mathrm{Pc}=\frac{2 \gamma \cos \theta}{\mathrm{R}}
$$

According to Eq. 1, theory presents that there are two limiting cases for the penetration of a droplet into a porous material. In the first case, the contact line of the drop with the solid surface remains constant to its initial position, that is to say, keeping drawing area penetration as a constant, meaning that the contact angle decreases and that the radius of curvature of the drop increases during penetration process. In the second case, keeping the contact angle of the drop as a constant as it penetrates the bed, and the base area decreases with the reduction of volume, this is often referred to as the reducing drawing area case[7].

The spreading of droplet on the powder bed is driven by a competition between capillary driving forces and viscous dissipation, where the capillary forces is driven by the surface tension and viscous dissipation driven by the viscosity. This can be characterized by Tanner's Law, Eq. 2 and Eq. 3. where $d$ and $\theta_{D}$ are the spreading diameter and dynamic apparent contact angle respectively.

$$
\begin{aligned}
& d \propto t^{0.1} . \\
& \theta_{D} \propto t^{-0.3} .
\end{aligned}
$$

During the stage of nucleation, there are two important competing mechanism to quantify the effects of the most important process variables in the nucleation. One is the spray rate of granulation liquid and the re-entering spray zone covered by spray nozzle[8]. Here, we can define a dimensionless spray flux $\psi$ a, Eq. 4 , where V is the volumetric spray rate flowrate of spray nozzle, $\mathrm{d}$ is the average drop size, and the powder surface is traversing the spray zone with a flux A.

$$
\psi_{a}=\frac{3 V}{2 A d}
$$

The other one is the penetration rate of granulation liquid. We can define another dimensionless drop penetration time $\tau p$, Eq. 5, where tp is the penetration time of the spray drops, $t \mathrm{t}$ is the circulation time, which is the time interval between a packet of powder leaving and re-entering the spray zone.

$$
\tau_{p}=\frac{t_{p}}{t_{t}}
$$

The dimensionless spray flux and drop penetration time propose nucleation regime map (Figure 1 ) . There are three regimes in the map, they include drop controlled regime, intermediate regime and mechanical dispersion regime. Ideal nucleation conditions occur in the drop controlled regime. In this regime, one droplet almost makes one nuclei which tends to form one granule, because the spray droplets penetrate quickly into the powder and are well dispersed. In order to obtain ideal nucleation conditions in the drop controlled regime, two requirement must be satisfied: lower $\psi \mathrm{a}$ and quicker $\tau p$. That is to say, (1) The powder flux through the spray zone fast to avoid that droplets overlap. (2) The powder bed must be wetted by the droplet completely before powder bed mixture brings the droplet into contact with another partially absorbed droplet on the powder bed surface. We can gain lower $\psi \mathrm{a}$ and quicker $\tau \mathrm{p}$ via controlling operating conditions and particular formulation properties respectively[9]. In Figure.1, the process assumes that only dry powder enters the spray zone. However, in pharmaceutical industry, the partially wetted powder recirculates many times through the spray zone. The phenomenon of partially wetted powder reentering the spray zone would result in that one droplet overlaps with a nuclei formed on a previous pass through the spray zone. Hence, Hapgood proposed Nucleation regime map with the adjusted boundaries (Figure 2)[9].

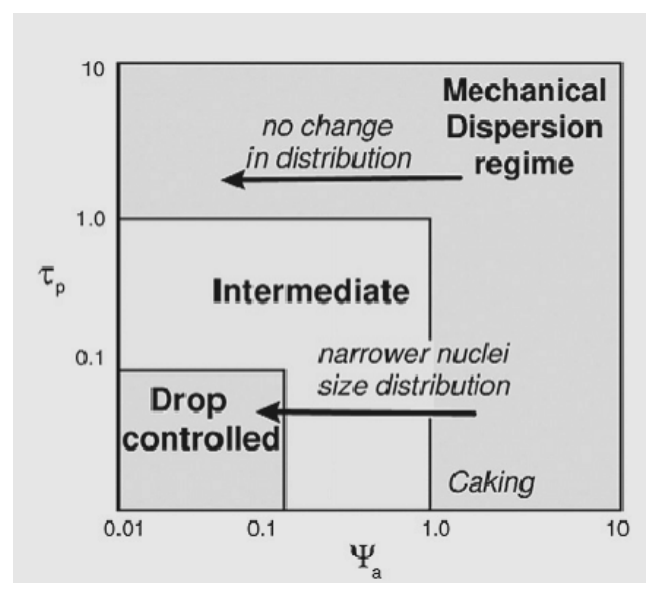

FIGURE I. NUCLEATION REGIME MAP[9]

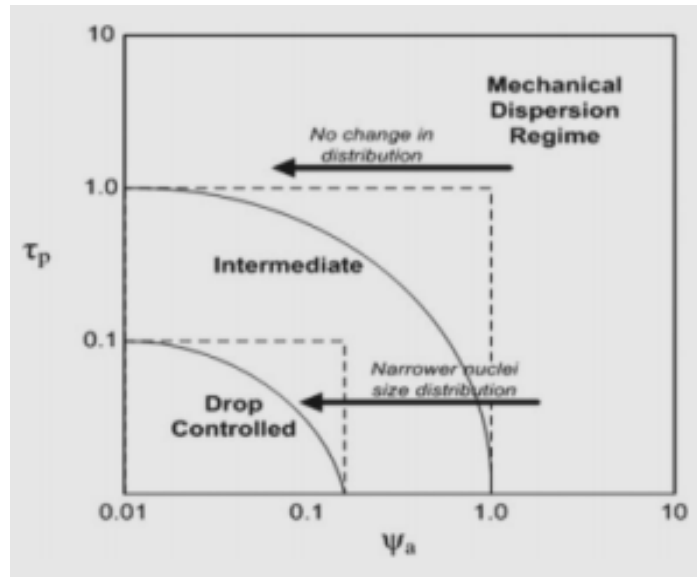

FIGURE II. NUCLEATION REGIME MAP WITH THE ADJUSTED BOUNDARIES [10] 
The nucleation regime map do be a useful guide to determine whether droplet controlled nucleation would occur, but it can't predict the structure and morphology of final granules, it can't also describe the details of the mechanisms that granules are formed. The nucleation regime map pay the most attention to the particle size and particle size distribution as these are the easiest properties to measure. However, other properties of granules, such as porosity, shape, and internal structure, are also play important role in dictating predicting and controlling properties of final granule. Therefore, Emady proposes three different granule formation mechanisms: Tunneling, Spreading, and Crater Formation[11]. For loose and cohesive powder beds, granules are formed by a Tunneling mechanism. In this case, powder aggregates would be sucked into the droplet which then tunneled into the powder beds. Spreading occurs for coarser powders at a low impact velocity. While Crater Formation occurs at a high impact velocity, the droplet would form a crater on surface of the powder bed and deform elastically in the crater. And the droplet would be coated in a layer of powder before penetrating into the bed by capillary action. The different granule shapes observed can be explained by the three different granule formation mechanisms. Round granules are always produced by Tunneling mechanism, flat disks are always produced by the Spreading mechanism, and Crater Formation mechanism produces granules with varying shapes that are depended on properties of liquid binder.

\section{CONSOLIDATION AND GROWTH}

The dimensionless Stokes deformation number $\left(S t_{d e f}\right)$ Eq. 6 and the maximum granule pore saturation $\left(S_{\max }\right)$ Eq. 7 are usually used to characterize the ratio of impact kinetic energy to the plastic energy absorbed per unit strain. Since the soluble components can dissolve gradually and granule pore liquid saturation will vary during the process of consolidation in granulation, $S_{\max }$ can be used as the measure of liquid content[9]. $S t_{d e f}$ is the ratio of impact kinetic energy to the plastic energy absorbed per unit strain[12].

$$
S t_{d e f}=\frac{\rho U^{2}}{2 Y} .
$$

Where $\rho$ is the granule density, $U$ is the representative collision velocity in the granulator, $Y$ is dynamic yield stress.

$$
S_{\max }=\frac{W \rho_{s}\left(1-\varepsilon_{\min }\right)}{\rho_{l} \varepsilon_{\min }} .
$$

Where $\rho_{s}$ and $\rho_{l}$ are the density of the solid particles and the liquid density, respectively. $W$ is the mass ratio of liquid to solid, and $\varepsilon_{\min }$ is the minimum porosity the formulation reaches for that particular set of operating conditions.

$S_{\max }$ and $S t_{d e f}$ can be plotted on a granule growth regime map[13]. On this occasion, we presume that particles spread uniformly on powder bed. It's noted that ideal nucleation conditions occur in the drop controlled regime.
Le etal[14] added liquid binder with two different colors into the powder bed, and obtained two kinds of granule structures. In their research, It was demonstrated there was a significant inhomogeneity in the granule properties, even for granules of the same size, in the same batch, granulated for the same amount of time. It was found that two parallel mechanism existed in granulation.

Bouwman etal[15] investigated the different mechanisms of material exchange during the equilibrium phase in granulation. They showed that three different mechanisms of material exchange existed: exchange by disintegration, exchange by deformation, and exchange by distribution. (1) The disintegration mechanism, where granules are crushed rapidly and formed to granules again, occurs during the process when granules are crushed to fragments upon collision with the impeller. As this fragmentation is followed by that the fragments re-agglomerate immediately to form new granules, this mechanism will lead to a complete and fast distribution of the tracer over all granules. (2) The deformation mechanism, where abraded granule fragments fuse with other granules immediately, occurs when the granules show abrasion or deformation upon impact and shear. While in a deformation, a part of the granule will break off from the main granule body. After that, the abraded parts will coalesce with another granule immediately, and be followed by re-arrangement to a sphere. This process happens too fast to observe fragments. (3) The distribution mechanism, where there is a prolonged period over which both tracer and standard granules stay intact, followed by uncontrolled growth and exchange of material, occurs when granules do have a very low densification rate. In this mechanism, growth hardly occurs after nucleation, it is possible that particle size has not changed for the slow granule growth rates and that long induction times equilibrium has not been reached yet when the tracer granules are added. While, the process will become uncontrollable (ball growth can be observed) when eventually densification occurs. Bouwman etal[15] also found that it's possible to shift among these mechanism by changing the process conditions.

\section{BREAKAGE AND ATTRITION}

Although granulation is a process of size enlargement, the emergence of breakage and attrition leads to the reduction of particle size and makes granulation products un controlled. However, granulation can make granules rounder, and promote the uniformity of granules[16].

The granule breakage and attrition behavior can be characterized by granule strength. It's defined as the resistance of the material to permanent deformation and fracture during a stressing event. Granule strength is dependent on varied bonding forces among granules: (1) Forces due to immobile films. On the surface of primary particles, a thin immobile liquid layer can be formed as the granule reaching a critical level of compaction or excess binder removal through evaporation. This strength is dependent on the contact area and the properties of the binder, such as the tensile strength of the liquid layer. (2) Forces due to mobile liquid bridges. It's mainly affected by liquid content. (3) Forces due to solid bridges. It's can be formed due to reasons such as 
crystallisation, chemical reactions, binder solidification and sintering. (4) Forces due to attractive effects between solid particles. It can take many different forms, such as van der Waals, magnetic and electro-static. (5) Forces due to mechanical interlocking between solid particles. Particles with irregular shape are packed tightly and contribute to granule strength significantly[17]. The granule strength can be characterized by yield strength, shear strain, tensile strength, bending strength and hardness of granules.

\section{SUMMARY}

As to the process of granulation, researchers have promoted multifarious models and mechanisms that are useful to control the quality of granules. Quality by Design (QbD), which is different from traditional Quality by Testing (QbT), is a semi-empirical approaches, which is at the intermediate level between the extremes of data-driven empirical models and first principles models. This model is aimed at establishing a relationship among original material, formulation design and granulation process parameters, Although not fully predictive, regime maps and dimensionless parameters are particularly useful for predicting the properties of final granules[18]. Now, we can make a study on formulation properties and process operation parameters with using particle design by implementation of the process of HSG, and predict the result of granulation. Based on the applying of advanced technologies and processes, improved formulation properties as well as operating parameters and equipment, a good prediction ability and accurate controlling in case of final granule product could be accomplished.

The author believes that the future development of HSG would follow some principles. Primarily, making further study on mechanism of granulation, increasing the introduction of interdisciplinary subjects and illustrating the mechanism of granulation aim to promote the adaptability of models to complex system. While mechanism of granulation can be illustrated artificial neural network, Fuzzy Mathematics theory, population balance modeling. Posteriorly, it's important to strengthen the research on the transmission law of physical properties. Q8 guidelines encourage researchers to pay more attention to online measurement and interrelated properties which affect the granulation products significantly. That is to say, researchers can use Process Analytical Technology (PAT). Therefore, it's very vital to speed up the transmission of physical properties from wet granules to dry granules, it's also important to strengthen the study on the rheological characteristic of wet granules. In order to obtain ideal granules, researchers should pay more attention to the research on the transmission law of physical properties from powder to granules, formulation preparation and improvement on the properties of excipients or process operation parameters.

\section{ACKNOWLEDGMENT}

The work was supported by the grant of the National Natural Science Foundation of China (No. 81403102).

\section{REFERENCES}

[1] M. Börner, M. Michaelis, E. Siegmann, C. Radeke, U. Schmidt, "Impact of impeller design on high-shear wet-granulation," Powder Technol, Vol. 295, pp. 261-271, July 2016.
[2] P. Kulinowski K.W. Orlewicz, J. Obral, GM Rappen, D Haznar-Garbacz, et al, "Multimodal approach to characterization of hydrophilic matrices manufactured by wet and dry granulation or direct compression methods,” Int. J. Pharm. Vol. 499, pp. 263-270, February 2016.

[3] D.A. Pohlman, and J.D. Litster, "Coalescence model for induction growth behavior in high shear granulation,” Powder Technol. Vol. B270, pp. 435-444, January 2015.

[4] C. Mangwandi, M.J. Adams, M.J. Hounslow, and A.D. Salman, "Effect of batch size on mechanical properties of granules in high shear granulation," Powder Technol. Vol. 206, pp 44-52, January 2011.

[5] E. Franceschinis, A.C. Santomaso, L. Benda, B. Perissutti, D. Voinovich, et al, "Influence of process variables on the properties of simvastatin self-emulsifying granules obtained through high shear wet granulation," Powder Technol. Vol. 274,pp 173-179, April 2015.

[6] K. Ax, H. Feise, R. Sochon, M. Hounslow, and A. Salman, "Influence of liquid binder dispersion in agglomeration in an intensive mixer," Powder Technol. Vol. 179, pp 190-194, January 2008.

[7] H.R. Charles-Williams, R. Wengeler, K. Flore, H. Feise, M.J. Hounslow, et al, "Granule nucleation and growth: competing drop spreading and infiltration process," Powder Technol. Vol. 206, pp 63-71, January 2011.

[8] J.D. Litster, K.P. Hapgood, J.N. Michaels, A. Sims, M. Robert, et al, "Liquid distribution in wet granulation: dimensionless spray flux," Powder Technol. Vol. 114, pp 32-29, January 2001.

[9] S.M. Iveson, J.D. Litster, K. Hapgood, and B.J. Ennis, "Nucleation, growth and breakage phenomena in agitated wet granulation processes: a review," Powder Technol. Vol. 117, pp 3-39, June 2001.

[10] K.P. Hapgood, T.H. Nguyen, H. Sunarko, S.M. Lveson, and S. Wei, "Rewetting effects and droplet motion on partially wetted powder surfaces," Aiche J. Vol. 55, pp 1402-1415.

[11] H.N. Emady, D.K. Talay, W.C. Schwerin, and J.D. Lister, "Granule formation mechanisms and morphology from single drop impact on powder beds," Powder Technol. Vol. 212, pp 69-79, September 2011.

[12] G. Tardos, M. Khan, and P. Mort, "Critical parameters and limiting conditions in binder granulation of fine powders," Powder Technol. Vol. 94, pp 245-258.

[13] S.M. Iveson, P.A.L. Wauters, S. Forrest, J.D. Lister, G.M.H. Meesters, et al, "Growth regime map for liquid-bound granules: further development and experimental validation," Powder Technol. Vol. 117, pp 83-97, June 2001.

[14] P.K. Le, P. Avontuur, M.J. Houunslow, and A.D. Salman, "A microscopic study of granulation mechanisms and their effect on granule properties, " Powder Technol. Vol. 206, pp 18-24, January 2011.

[15] A.M. Bouwman, M.R. Visser, G.M.H. Meesters, and H.W. Frijlink, "The use of Stokes deformation number as a predictive tool for material exchange behavior of granules in the 'equilibrium phase'in high shear granulation,'Int. J. Pharm. Vol. 318, pp 78-85, August 006.

[16] K. Dries, O.M. Vegt, V. Girard, and H. Vromans, "Granule breakage phenomena in a high shear mixter; influence of process and formulation variables and consequences on granule homogeneity," Powder Technol. Vol. 133, pp 228-236, June 2003.

[17] G.K. Reynolds, J.S. Fu, Y.S. Cheong, M.J. Hounslow, and A.D. Salman, "Breakage in granulation: a review," Chem. Eng. Sci. Vol. 60, pp 3969-3992.

[18] D. Kayrak-Talay, S. Dale, C. Wassgren, and J. Lister, "Quality by design for wet granulation in pharmaceutical processing: assessing models for a priori design and scaling," Powder Technol. Vol. 240,pp 7-18, May 2013. 